\title{
Spontaneous intramural rupture and intramural haematoma of the oesophagus*
}

\author{
W F KERR \\ From the Norfolk and Norwich Hospital, Norwich
}

ABSTRACT Spontaneous intramural rupture or intramural haematoma of the oesophagus is rare cause of acute pain in the chest and upper abdomen. Much less ominous than spontaneoug complete rupture from which it must be distinguished, it seldom if ever necessitates operation. Five new cases are described and reviewed together with 15 collected from published reports The dominant symptom of every case was severe and constant retrosternal or epigastric pain concomitant dysphagia was mentioned in 11 cases. In seven the pain was preceded by or cos incided with vomiting. The condition was related to other stresses in three and appeared to be truly spontaneous in 10 . In approximately one-third of cases it started suddenly but more ofte $\vec{P}$ it began as discomfort worsening rapidly. Fourteen patients vomited blood after experiencing pain but only four were given transfusions. In contradistinction to complete rupture, none ha\& surgical emphysema and plain chest radiographs were unremarkable. All had abnormal gastros grafin or barium swallows. Intramural haematomas with or without mucosal tears were seen i the 11 cases in which oesophagoscopy was performed. Fifteen patients made rapid and complete recoveries on conservative management. Of the four who did not respond satisfactorily, one had the oesophagus repaired, two had drainage of the mediastinum after failure to find the false lumen at thoracotomy, and one had only an abdominal exploration. The only death in the whole series occurred after a disastrous emergency exploration and subsequent total oesophagectomy.

The first editor of Thorax wrote for the first issue of this journal the article that was largely responsible for transforming spontaneous perforation or rupture of the oesophagus, the Boerhaave syndrome, from a little-known necropsy finding to a generally recognised clinical entity. ${ }^{1}$ After describing the symptoms and signs by which the diagnosis could and should be made during life, Barrett argued that immediate repair was the logical method of treatment and offered the best hope of survival, and a year later he himself had the satisfaction of proving his thesis by recording the first succesful operation for that catastrophic condition. ${ }^{2}$ While there can be few nowadays who would recomend conservative management for the Boerhaave syndrome diagnosed early, sporadic reports have, since 1957, drawn attention to a

*Based on a paper read to the Society of Thoracic and Cardiovascular Surgeons of Great Britain and Ireland, Lausanne, September 1980.

Address for reprint requests: WF Kerr, Norfolk and Norwich Hospital, Brunswick Road, Norwich NR1 3SR. partial or intramural type of oesophageal rupture in which surgical intervention is seldom if eve? required. It is, therefore, important to differentiate spontaneous intramural rupture from the much more serious complete or transmural rupture oro the one hand, and from the numerous causes of acute pain in the chest and upper abdomen or the other.

Mucosal ruptures causing intramural haema toma and abscess formation may be produced bs swallowed foreign bodies or by instrumentation ${ }^{3-6 . \mathrm{W}}$ they may also occur "spontaneously." A compute search of published reports has brought to light only 15 examples of spontaneous intramuraf rupture, ${ }^{5-14}$ and those include one case attributed. by the author to remote trauma and six reportedo variously as submucosal dissection caused b. haemorrhage, dissecting haematoma, and oeso@ phageal apoplexy. The five new cases describe $\mathbb{B}$ below illustrate the principal features and the problems of this syndrome. 


\section{Definitions}

"Rupture" and "perforation" are used synonymously. "Haematoma" and "dissection" emphasise developments associated with mucosal tears. Basically the terms all refer to the same pathological process. Several articles about rupture or perforation mention haematomas seen at oesophagoscopy or imagined as explanations of radiological appearances, while authors writing about haematomas and dissections describe or postulate mucosal rupture to account for blood in the oesophageal lumen and radiological contrast material outside it. "Spontaneous" is used in the sense that has become conventional in oesophageal surgery, acknowledging that in many instances it is neither accurate nor appropriate.

\section{Case reports}

Unless otherwise stated, in all five cases on first admission to hospital, physical examination produced no specific or characteristic findings, electrocardiographs and plain chest radiographs were unremarkable, and routine laboratory estimations were within normal limits.
CASE 1

This 65-year-old woman (IG) had for many years suffered from "indigestion," usually relieved by antacids. In January 1973, feeling she had eaten too much, she forced herself to vomit and, as she had done once before in similar circumstances, she brought up a small quantity of blood. A barium swallow and meal soon afterwards was reported as normal. On the evening of 4 November 1976, she took a meal of mince and mashed potatoes and was eating an apple when she began to feel retrosternal discomfort and "knew from experience that she was going to be sick." A little later she found that she could not swallow, and that was a symptom new to her. She took some bicarbonate of soda and tried to force herself to vomit but did not even retch. The discomfort rapidly became continuous pain, greatly aggravated by swallowing saliva. Approximately 40 hours after first complaining, she was admitted to an ear, nose, and throat ward and transferred to the Thoracic Surgical Unit a few hours later, after a barium swallow, taken with great reluctance and very obvious distress, had shown a filling defect in the lower oesophagus (fig 1). Oesophagoscopy disclosed blood in the lumen of the oesophagus and an intramural haematoma extend-

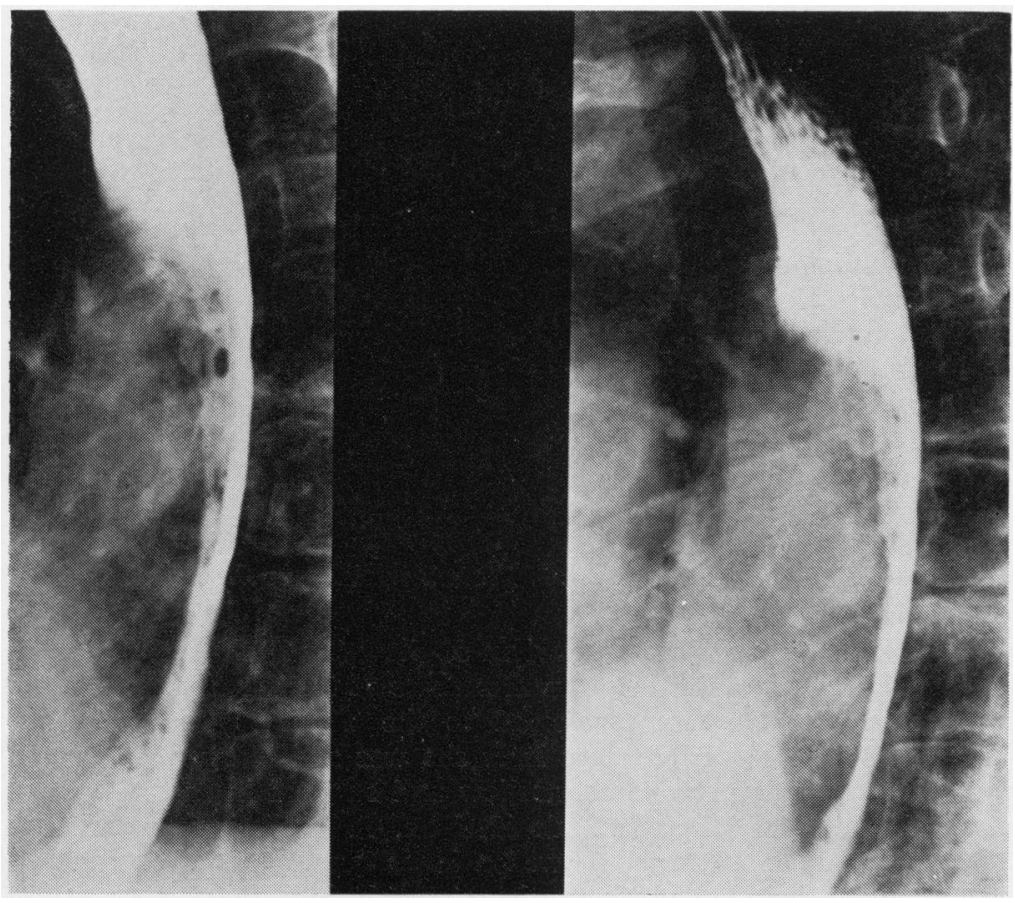

Fig 1 Case 1. Barium swallow cn the third day of complaint. 
ing from $29 \mathrm{~cm}$ from the upper alveolar margin to $38 \mathrm{~cm}$, with an acute ulcer or mucosal tear on the posterior wall at the lower end. She was fed through a nasogastric tube for three days and her symptoms rapidly disappeared. A barium swallow on the ninth day after admission showed the lower oesophagus slightly distorted but no longer obstructed. At oesophagoscopy the next day the only abnormality was a dusky and rather friable mucous membrane between 25 and $31 \mathrm{~cm}$.

Two years later, she again began to experience central chest pain on swallowing and a sensation of food sticking in her gullet and she lost $6.4 \mathrm{~kg}$ $(14 \mathrm{lb})$ in weight in four months. New barium swallows showed diffuse narrowing of the lower oesophagus which would occasionally open out to a lumen of adequate size. Oesophagoscopy was normal but oesophageal manometry revealed gross incoordination of muscular activity in the lower half of the oesophagus. At thoracotomy on 18 April 1979 the lower oesophagus was found to be extremely thin, almost hypoplastic; there was no other obvious abnormality. An extended oesophagomyotomy was carried out, a plane of cleavage between the muscular and submucous layers being developed easily in the lower $5 \mathrm{~cm}$ but with difficulty above, to a level well into the apparently normal upper segment. Histological examination of a biopsy showed "no recognisable abnormality in the muscle layers, ganglion cells, or autonomic nerve fibres." After this operation the patient has remained well except for a minor recurrence of dysphagia which remitted spontaneously within a fortnight.

\section{CASE 2}

A woman (BL), under treatment for pernicious anaemia and myxoedema, was exceptionally alert and vigorous for her 77 years and had enjoyed a round of golf two days before she became ill. On 19 October 1978 she took her evening meal as usual without incident. Soon afterwards she began to feel pain across the front of her chest. She went early to bed but wakened at 2 am with a rasping cough and pain so severe that she had to send for her doctor. The pain was constant and radiated up to the right side of her neck and down to the epigastrium. There was no history of nausea, vomiting, indigestion, or postural regurgitation. She was treated at home with antacids and analgesics but the pain got worse, and she was admitted to a general surgical unit on 23 October. Although she did not complain of difficulty in swallowing, she would not take food or fluids because they aggravated the pain and made her

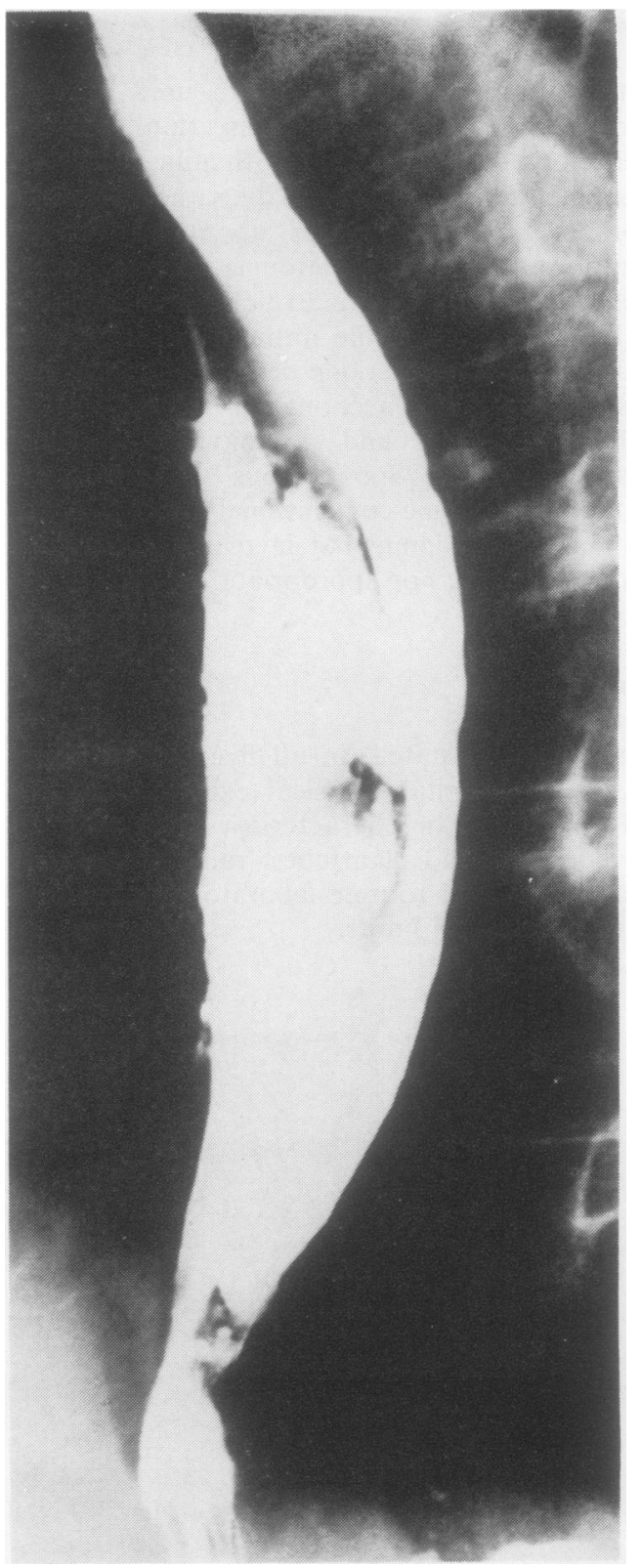

Fig 2 Case 2. Barium swallow on the fourth day.

vomit. A barium swallow (fig 2) showed an exten sive intramural rupture of the oesophagus and she was transferred to the Thoracic Surgical Unit. Three days later she was still in severe pain and unable to swallow and surgical intervention was? therefore, decided on. A preliminary oesophag $\cong$ oscopy showed a large quantity of fluid regurgi tated from the stomach into the pharynx and 
upper oesophagus. From $25 \mathrm{~cm}$ from the upper alveolar margin onwards, the mucous membrane was blue and bulged inwards. At $32 \mathrm{~cm}$ there was a transverse laceration of the posterior and right wall of the oesophagus; no blood, pus, or barium could be seen exuding from it. The remainder of the oesophagus was normal except for the discoloration which extended to the cardia. When the chest was opened through a right thoracotomy, there was no pleural effusion and the lung was normal. The mediastinal pleura from the thoracic inlet to the diaphragmatic hiatus was stained with old blood, most intensely at the level of the azygos vein. The pleura was incised; there was no evidence of mediastinal soiling beyond that already mentioned. A discoloured and considerably thickened oesophagus was mobilised but a prolonged search failed to locate either the plane of dissection or the mucosal tear without incising the mucous membrane. The chest was closed with drains to the mediastinum and a jejunostomy was established for feeding. The patient made a slow but complete recovery.

\section{CASE 3}

This woman (KD), aged 80 years, had frequently complained of "indigestion" and had also been treated for ischaemic heart disease for a number of years. On 1 August 1979 she was watching her village carnival when she was seized with "crushing" central chest pain which radiated to her neck. At the same time she complained of a lump in her throat and that swallowing was painful. There had been no nausea, retching, or vomiting. She was given an injection of diamorphine and perchlorperazine and admitted immediately to a nearby geriatric unit, where a fresh myocardial infarct was quickly excluded. By the next morning her pain had become much less severe but its aggravation by swallowing was more pronounced. She was treated with antacids and continued to improve, taking liquids and solids reasonably well but complaining constantly of the "lump" in her throat. A barium swallow on the eighth day revealed an intramural rupture of the oesophagus (fig 3 ). The conservative regime was continued and the patient made a satisfactory recovery. A month later, another barium swallow showed no sign of the rupture but suggested early achalasia. No more investigations were considered necessary.

\section{CASE 4}

A woman of 59 years (SK) was in her normal good health when she sat down to lunch on 22 March

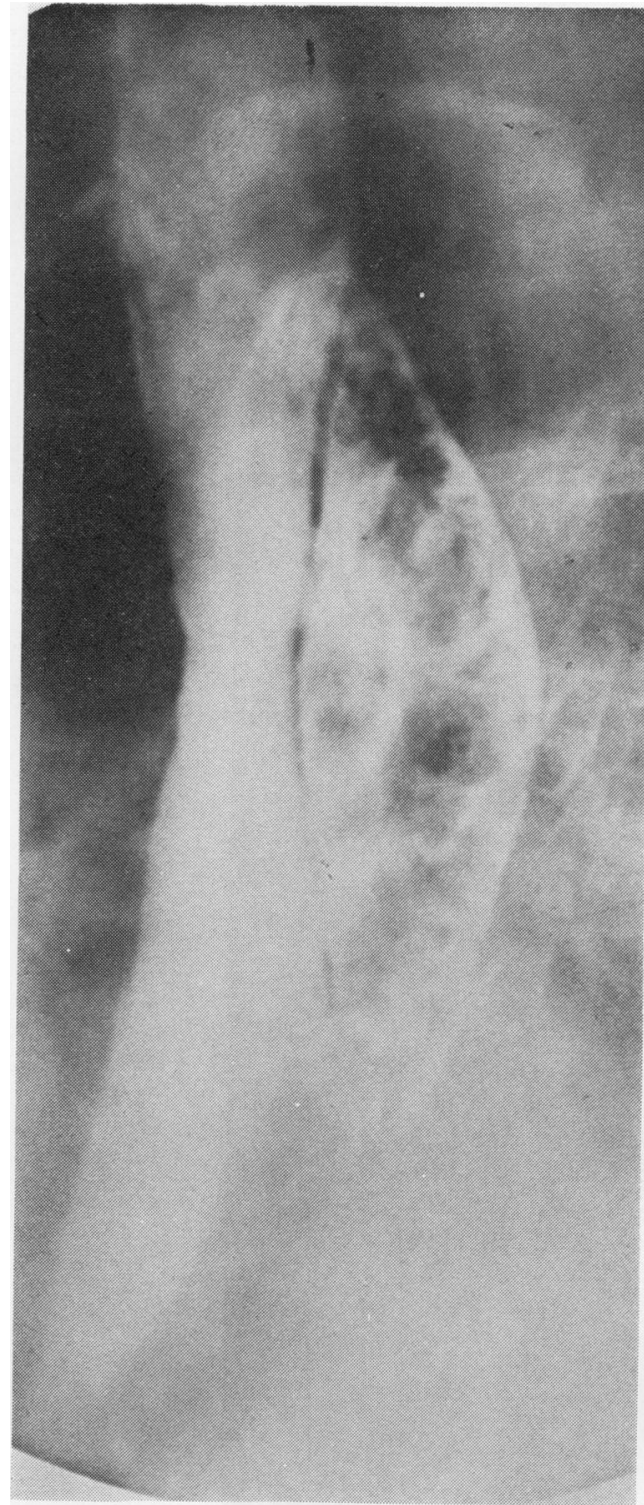

Fig 3 Case 3. Barium swallow on the eighth day.

1980. She had taken only one mouthful of freshlycooked tender green beans when she experienced severe burning pain retrosternally and in the epigastrium, continuous and made worse by swallowing. It was not preceded by nausea, retching, or vomiting but after about 20 minutes she vomited a small quantity of fresh blood and called her doctor. While he was examining her she vomited altered blood. She was admitted as an emergency to a general medical ward, a nasogastric tube was passed without difficulty, and 
normal gastric secretions were aspirated. A conservative regime for haematemesis was instituted and by the next morning she felt better, had pain unly on swallowing, and had had no more haematemesis, but her haemoglobin had dropped from 15.2 to $11.2 \mathrm{~g} / \mathrm{dl}$. Next day she had a slight rise in temperature and a new chest radiograph showed a small left pleural effusion. A barium examination on the fourth day (fig 4) demonstrated an intramural rupture just above a sliding hiatal hernia. The patient was transferred to the Thoracic Surgical Unit where oral feeding was

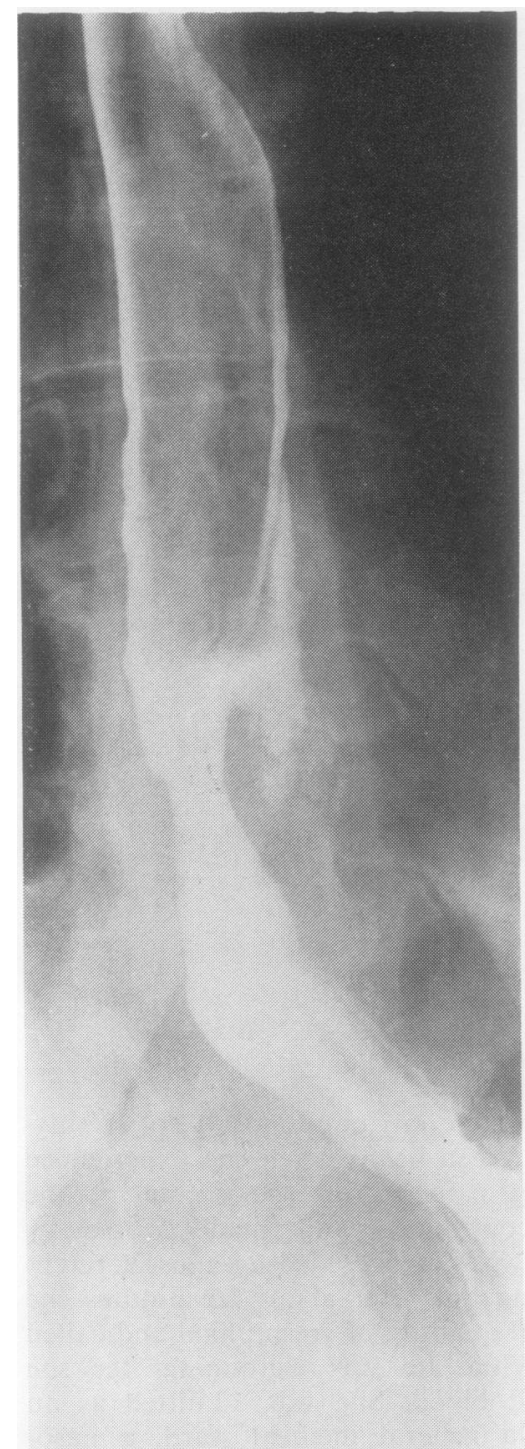

Fig 4 Case 4. Barium swallow on the fourth day. discontinued. Within a week she was symptom $-\frac{0}{0}$ free and a barium swallow on the eleventh days showed that the oesophageal rupture had healed. $\varnothing$ In retrospect the patient admitted she had hado mild heartburn on and off for several months buts never severe enough to warrant a visit to her ${ }^{\circ}$ doctor.

\section{CASE 5}

This 72-year-old woman (EW), obese and hyper tensive, pain and dysphagia for several years, and she had had more than one minor episode of haema temesis. On 30 April 1977, without premonition, she vomited about a pint $(570 \mathrm{ml})$ of fresh blood and complained of severe epigastric pain. She was? admitted to a medical unit where the abdomen was noted to be soft but with tenderness localised? to the epigastrium. The next day, after blood transfusion, a fibreoptic oesophagoscopy was. carried out. There was fresh blood and blood-clozo in the upper oesophagus, the mucous membrane was haemorrhagic, and the oesophagus was obstructed at mid-oesophageal level. The radio graphic appearance after oesophagoscopy is showr

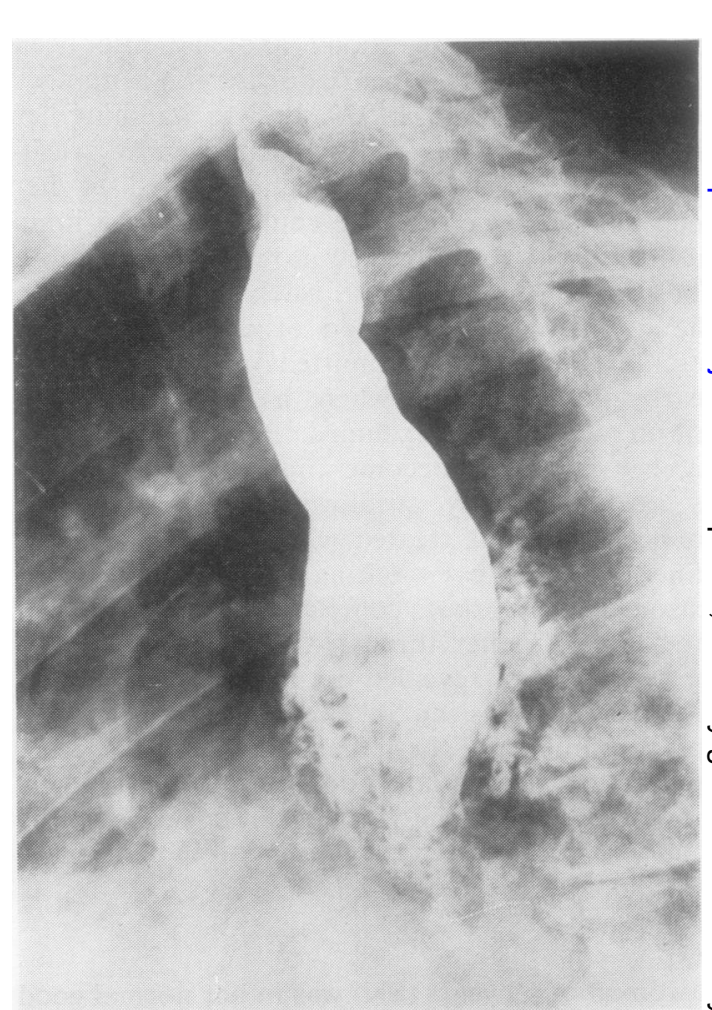

Fig 5 Case 5. Barium swallow on the second day. 
in fig 5. Next day after her transfer to a general surgical unit, the abdomen was explored. No explanation for the oesophageal abnormality was discovered; the passage of a large tube into the stomach was accepted as evidence that there was no significant obstruction of the oesophagus. Two weeks later after a postoperative course complicated by respiratory failure and a left pleural effusion, another barium swallow showed between the arch of the aorta and the diaphragm the

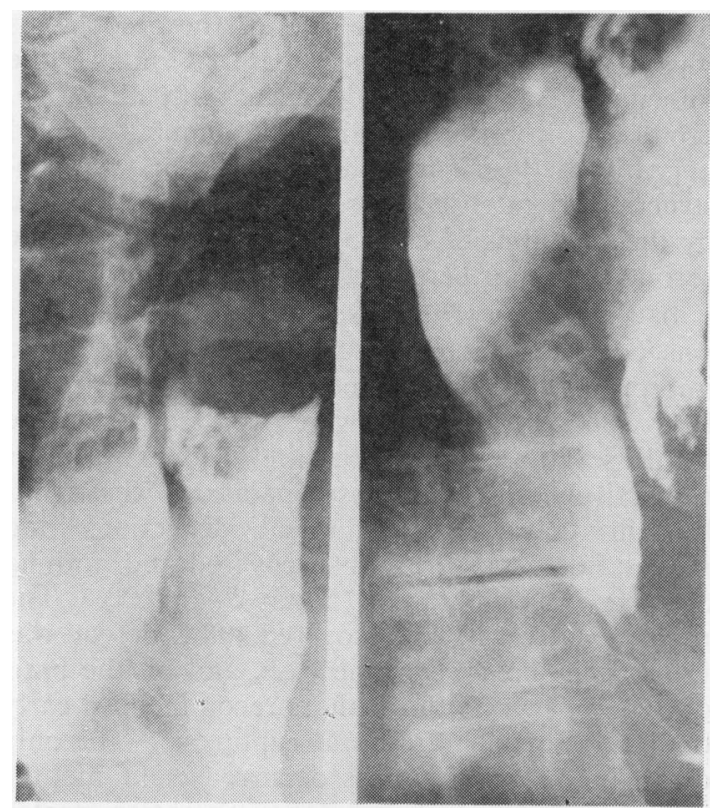

Fig 6 Ca:e 5. Barium swallow on the fourteenth day double-barrelled appearance of a dissecting haematoma (fig 6). When a cardiothoracic surgeon was consulted he recognised the condition and under his supervision the patient made a full recovery in six weeks without further surgical interference.

\section{Discussion}

It is tempting to assume that spontaneous intramural rupture of the oesophagus represents an intermediate stage between the lesions of the Mallory-Weiss and the Boerhaave syndromes but there is room for an alternative explanation. A review of the five cases reported here together with the 15 previously reported has shown that whereas in the Mallory-Weiss and Boerhaave syndromes there is a great preponderance of men and a strong causative link with vomiting, in spontaneous intramural rupture elderly women are most frequently affected (fig 7), and vomiting preceded or coincided with pain in only seven out of the 20 cases. There were other stresses capable of suddenly raising the intraoesophageal pressure in three more patients but in the remaining $50 \%$ including one receiving heparin, nothing extraordinary happened to provoke injury or haemorrhage. For them "spontaneous" seems a wholly appropriate adjective. Neither the Mallory-Weiss nor the Boerhaave lesions are associated with submucosal haematomas or dissections although there is the authority of Weiss and Mallory themselves for the statement that "their" lesions may penetrate as deep as the muscle layer. ${ }^{15}$ The tears in both are longitudinal,

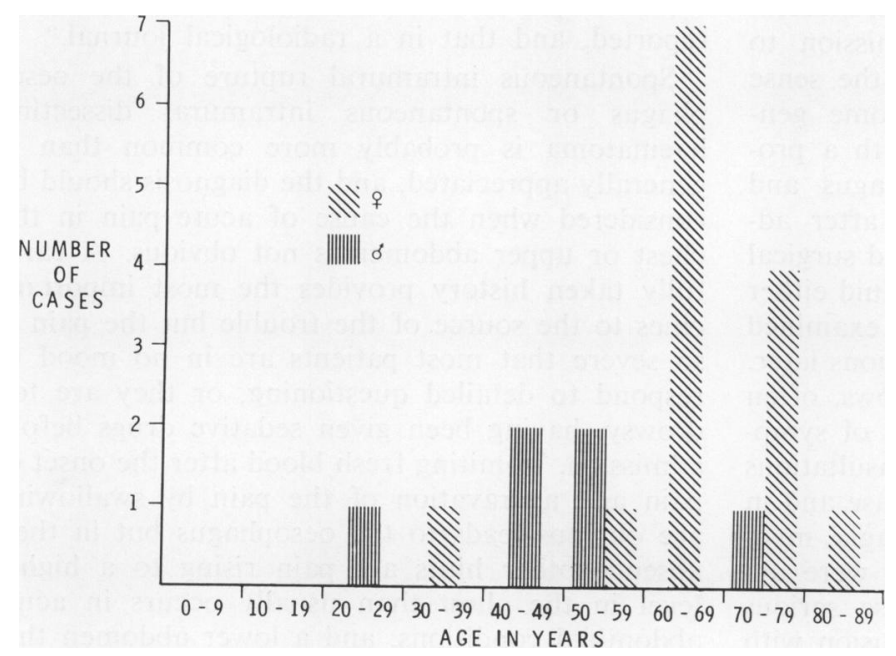

Fig 7 Age and sex incidence of 20 cases of spontaneous rupture of the oesophagus. 
close to the cardia in the former, in the lower oesophagus as a rule in the latter with the middle third only occasionally involved. ${ }^{16}$ The mucosal tears associated with spontaneous intramural ruptures were not confined to any one segment but were distributed throughout the length of the organ, and the only two described with precision at oesophagoscopy were transverse, while the one exposed at operation was longitudinal. There are therefore grounds for holding that in some cases the first event may be haemorrhage into the submucosa with secondary rupture into the lumen.

The clinical picture in every case was dominated by pain. Without exception it was described as severe, sometimes crushing, and several patients were said to be shocked on admission to hospital. It was felt mainly in the chest or upper abdomen and frequently radiated to the back, neck, and throat. Eleven patients $(55 \%)$ complained that swallowing made the pain worse but that symptom was sometimes overshadowed at first, and emerged into significance only when the pain in the chest was subsiding. That pain was reported to have started suddenly in seven cases; a more usual mode of onset was retrosternal discomfort building up rapidly to pain in the course of $20-30$ minutes, and this group included several of the cases attributed to vomiting. A history of antecedent dyspepsia was obtained from only six patients.

Fourteen patients $(70 \%)$ vomited blood after the onset of pain but only three in addition to the man receiving heparin were given blood transfusions. Generally the quantity of blood lost was small.

Despite the severity of the pain and its not infrequent association with haematemesis, medical attendants both before and after admission to hospital were clearly not imbued with the sense of urgency that the Boerhaave syndrome generates. No one was sent to hospital with a provisional diagnosis of ruptured oesophagus and nothing in the physical examinations after admission suggested that. No case exhibited surgical emphysema, pneumothorax, or pleural fluid either clinically or radiologically when first examined although a few did develop pleural effusions later. Only the appearances on barium swallows, often delayed for several days from the onset of symptoms, gave rise to anxiety and led to consultations with thoracic surgeons. In the acute phase and in the absence of haematemesis and dysphagia, myocardial infarction and aortic dissection were obvious considerations, but did not cause serious problems in differential diagnosis. Confusion with upper abdominal emergencies seems to have give the greatest difficulty and two patients had laparotomy even though it was known before $\stackrel{\mathbb{D}}{\mathscr{D}}$ hand that the oesophagograms were abnormal. number of cases were treated initially as "haematemesis" without a specific diagnosis despite the intensity of the pain.

The crucial investigations were radiologicas Plain films of the chest and abdomen disclosed n $\vec{\alpha}$ significant abnormalities but contrast studies werg్ distinctive. They demonstrated obstruction caused by the haematoma in the wall of the oesophaguso (figs 1,5 ) or else contrast material outside the lumen but closely applied as a strip alongsideo producing in some instances a double-barrelled appearance (figs 2-6). The progression from obE struction to extensive dissection is well illustrate by the two films of case 5 (figs 5,6 ) taken on the second and fourteenth days respectively.

In this series of 20 cases, the initial treatment was conservative in 19, and it resulted in rapiळo improvement and complete recovery in 15 . The four who failed to settle were operated on, on the third, seventh, fourteenth, and eighteenth days respectively. Three of these operations were fruito less in that the submucosal dissections and mucosal defects were not discovered and nothing curative was achieved (cases 2 and 5 ). ${ }^{7}$ Th $\vec{E}$ patients nevertheless recovered. The defect was found and repaired in one case, and on the basis of a single success the authors recommended early. operation for all intramural ruptures! ${ }^{12}$ The only death in the whole series occurred eight month after a misconceived emergency operation started in the abdomen and followed by total oesophagec 3 tomy at a time when only one case of intramuraक rupture, spontaneous or instrumental, had been reported, and that in a radiological journal. ${ }^{9}$

Spontaneous intramural rupture of the oeso phagus or spontaneous intramural dissectin haematoma is probably more common than is generally appreciated, and the diagnosis should bo considered when the cause of acute pain in the chest or upper abdomen is not obvious. A careస్ట fully taken history provides the most important clues to the source of the trouble but the pain is so severe that most patients are in no mood to respond to detailed questioning, or they are to\& drowsy, having been given sedative drugs before admission. Vomiting fresh blood after the onset of pain and aggravation of the pain by swallowing are obvious leads to the oesophagus but in theip absence other hints are pain rising to a highe 5 level in the chest than usually occurs in acutê abdominal conditions, and a lower abdomen thag 
is soft on palpation when the upper abdomen is rigid. The pain of chronic peptic ulceration tends to be relieved, not aggravated, when bleeding starts, and the Mallory-Weiss syndrome is painless or nearly so. The growing practice of endoscoping early all cases of upper gastrointestinal tract bleeding will decrease the likelihood of error, but if intramural rupture is suspected, the first investigation should be a gastrografin swallow, for the radiological findings will confirm or exclude the diagnosis. Oesophagoscopy is not necessary. Almost all patients will recover quickly if oral feeding is stopped and antibiotics are administeredand the surgeon's nerve holds.

I am indebted to Mr RJ Sellick, Mr GF Langley, Dr BV Payne, and Dr JW Paulley for referring cases 1-4 respectively, and to $\mathrm{Mr} \mathrm{BB}$ Milstein for permission to include case 5; to Dr RS Thorpe, Dr I Williams, Dr WB Evans, Dr RJ Godwin, and Dr JJ Flanagan for radiological investigations; to $\mathrm{Mr}$ BA Ross for the manometric studies in case 1 and Dr PF Roberts for the histological report; and to the Department of Medical Illustration, Norfolk and Norwich Hospital for the illustrations.

\section{References}

1 Barrett NR. Spontaneous perforation of the oesophagus. Thorax 1946; 1:48-70.

2 Barrett NR. Report of a case of spontaneous perforation of the oesophagus successfully treated by operation. Br J Surg 1947; 35:216-8.
3 Sanborn EB. Intramural abscesses of the esophagus: a complication of foreign bodies. $J$ Thorac Cardiovasc Surg 1960; 39:586-92.

4 Lichter I, Borrie J. Intramural oesophageal abscess. Br J Surg 1965; 52:185-8.

5 Benjamin B, Hanks TJ. Submucosal dissection of the oesophagus due to haemorrhage. A new radiographic finding. J Laryngol Otol 1965; 79:1032-8.

6 Fráter LL, Imre J, Horváth ÖP, Rózsa Z. Intramurale ösophagusperforationen. Fortschr Röntgenstr 1978; 129:708-12.

7 Borrie J, Sheat J. Spontaneous intramural oesophageal perforation. Thorax 1970; 25:294-300.

8 Williams B. Oesophageal laceration following remote trauma. Br J Radiol 1957; 30:666-8.

9 Thompson NW, Ernst CB, Fry WJ. The spectrum of emetogenic injury to the esophagus and stomach. Am J Surg 1967; 113:13-25.

10 Marks IN, Keet AD. Intramural rupture of the oesophagus. $\mathrm{Br} \mathrm{Med} J \mathrm{~J}$ 1968; 3:536-7.

11 Joffe N, Millan VG. Postemetic dissecting intramural hematoma of the esophagus. Radiology 1970; 95:379-80.

12 Kelley DL, Neugebauer MK, Fosberg RG. Spontaneous intramural esophageal perforation. J Thorac Cardiovasc Surg 1972; 63:504-8.

13 Smith G, Brunnen PL, Gillanders LA, Teo HS. Oesophageal apoplexy. Lancet 1974; 1:390-2.

14 McMyn JK. Spontaneous intramural oesophageal perforation. Australas Radiol 1977; 21:234-40.

15 Weiss S, Mallory GK. Lesions of the cardiac orifice of the stomach produced by vomiting. JAMA 1932; 98:1353-5.

16 Bates $M$. Pressure rupture of the mid-thoracic oesophagus. Br J Surg 1969; 56:327-31. 